\title{
Fish consumption prior to pregnancy and pregnancy outcomes in the National Birth Defects Prevention Study, 1997-2011
}

\author{
Renata H Benjamin ${ }^{1, *}$, Laura E Mitchell ${ }^{1}$, Mark A Canfield ${ }^{2}$, Adrienne T Hoyt $^{2}$, Dejian Lai ${ }^{3}$, \\ Tunu A Ramadhani ${ }^{2}$, Suzan L Carmichael ${ }^{4}$, Amy P Case ${ }^{2}$, D Kim Waller ${ }^{1}$ and the National \\ Birth Defects Prevention Study \\ 'Department of Epidemiology, Human Genetics and Environmental Sciences, University of Texas Health Science \\ Center at Houston (UTHealth) School of Public Health, 1200 Pressler Street, Houston, TX 77030, USA: ${ }^{2}$ Birth Defects \\ Epidemiology and Surveillance Branch, Texas Department of State Health Services, Austin, TX, USA: ${ }^{3}$ Department of \\ Biostatistics, UTHealth School of Public Health, Houston, TX, USA: ${ }^{4}$ Department of Pediatrics, Stanford University, \\ Stanford, CA, USA
}

Submitted 23 March 2018: Final revision received 15 August 2018: Accepted 5 September 2018: First published online 17 October 2018

\begin{abstract}
Objective: To evaluate the relationships between maternal fish consumption and pregnancy outcomes in a large, population-based sample of women in the USA. Design: We collected average fish consumption prior to pregnancy using a modified version of the semi-quantitative Willett FFQ. We estimated adjusted OR (aOR) and 95\% CI for associations between different levels of fish consumption and preterm birth ( $<37$ weeks), early preterm birth $(<32$ and $<35$ weeks) and small-for-gestational-age infants (SGA; <10th percentile).

Setting: The National Birth Defects Prevention Study (NBDPS).

Subjects: Control mother-infant pairs with estimated delivery dates between 1997 and 2011 ( $n$ 10 919).

Results: No significant associations were observed between fish consumption and preterm birth or early preterm birth ( $\mathrm{aOR}=0 \cdot 7-1 \cdot 0$ and $0 \cdot 7-0 \cdot 9$, respectively). The odds of having an SGA infant were elevated ( $\mathrm{aOR}=2 \cdot 1 ; 95 \% \mathrm{CI} 1 \cdot 2,3 \cdot 4$ ) among women with daily fish consumption compared with women consuming fish less than once per month. No associations were observed between other levels of fish consumption and SGA (aOR $=0 \cdot 8-1 \cdot 0)$.

Conclusions: High intake of fish was associated with twofold higher odds of having an SGA infant, while moderate fish consumption prior to pregnancy was not associated with preterm or SGA. Our study, like many other studies in this area, lacked information regarding preparation methods and the specific types of fish consumed. Future studies should incorporate information on nutrient and contaminant contents, preparation methods and biomarkers to assess these relationships.
\end{abstract}

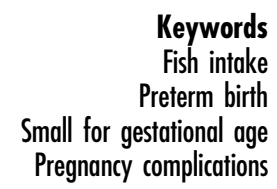

Keywords

Fish intake Small for gestational age Pregnancy complications
Fish, including both freshwater and saltwater fish and shellfish species, provide high-quality protein and nutrients, including long-chain $n-3$ PUFA (DHA and EPA) ${ }^{(1-3)}$. DHA and EPA are important for fetal neural and retinal development and they may reduce inflammatory processes, increase vasodilation, reduce platelet aggregation and influence the onset of labour through prostaglandin synthesis ${ }^{(1,2,4)}$. However, fish may also contain contaminants, such as methylmercury and persistent organic pollutants, which may adversely affect fetal development and impact pregnancy outcomes ${ }^{(5)}$.

Preterm and small-for-gestational-age (SGA) infants are at increased risk of morbidity, mortality and long-term developmental deficits ${ }^{(6,7)}$. Growth restriction and preterm delivery can sometimes be attributed to known causes, such as medical conditions and gestation of multiples; however, the aetiology remains unclear in many cases and novel approaches to prevention are needed. Recent studies $^{(8-15)}$ suggest that consuming fish during pregnancy may increase birth weight and help to protect against preterm birth; however, concerns remain about the effects of contaminants found in fish on fetal health. These concerns are heightened by several recent studies that have reported associations between high consumption of specific types of fish and increased risk of preterm birth or $\mathrm{SGA}^{(16-18)}$. 
Most of the studies that have assessed the associations between fish intake and pregnancy outcomes have been conducted in coastal European countries (Norway, Spain, France and Denmark), where fish consumption habits are different from those in the USA ${ }^{(9,10,13-17)}$. Per capita fish consumption in Norway is twice as high as that in the USA and inland farmed fish, including tilapia and catfish, make up a larger proportion of the domestic fish supply in the USA than in Europe ${ }^{(19)}$. Prior US studies examining the relationships between fish consumption and birth outcomes have focused on specific populations: one was a clinical trial of women at high risk for preterm birth ${ }^{(11)}$ and two were cohorts of predominantly non-Hispanic white women (66-88\%) living in specific geographic regions (Boston and Washington State) ${ }^{(18,20,21)}$. Results from European studies and prior US studies may not generalize to the US population as a whole.

The objective of the present study was to evaluate associations between fish consumption and preterm birth or SGA in a diverse sample of US women. To do so, we used data from the National Birth Defects Prevention Study (NBDPS), which surveyed a population-based sample of women as part of a case-control study of birth defects. Only control participants, who delivered an infant without a major structural birth defect, were included in the current analyses. To our knowledge, ours is the largest US study to examine the relationship between maternal fish intake and preterm birth and SGA.

\section{Methods}

\section{Population and design}

The NBDPS was a multisite, population-based, case-control study of birth defects with ten participating sites across the USA (Arkansas, California, Georgia/Centers for Disease Control and Prevention, Iowa, Massachusetts, New Jersey, New York, North Carolina, Texas and Utah) ${ }^{(22)}$. Controls were unmatched, live-born infants without a major birth defect, randomly selected from hospital records or vital records, who were born during the same time period and from the same geographic area as cases. Mothers were interviewed via a computer-assisted telephone interview between 6 weeks and 2 years after delivery $^{(22)}$. Interviews were conducted between 1997 and 2013 for infants with estimated due dates between 1997 and 2011. Participation rates were $67 \%$ among case mothers and $65 \%$ among control mothers.

The NBDPS used a shortened version of the semiquantitative Willett FFQ developed for the Nurse's Health Study to collect information on average maternal diet in the year prior to pregnancy, including information on how often women ate a 85-140 g (3-5 oz) serving of fish ${ }^{(23)}$. The questionnaire had sixteen possible responses for the frequency of fish consumption, ranging from never/less than once per month to six times or more per day.
The NBDPS maternal interview collected information about the infant including sex, date of birth and due date. For our primary analysis, preterm delivery was defined as a birth occurring at less than 37 weeks of gestation. For additional sensitivity analyses, early preterm birth was defined as a delivery occurring before 35 weeks of gestation and before 32 weeks of gestation. Since this was a secondary analysis of an existing study, we had limited power to evaluate early preterm delivery. However, since research suggests that associations between fish or fish oil consumption and preterm birth may differ between early and late preterm birth ${ }^{(14)}$, we sought to evaluate whether the associations between fish consumption levels and preterm birth differed when restricting preterm to earlier gestational ages. Infant birth weight was collected from medical records or birth certificates. SGA was defined as an infant with a birth weight below the 10th percentile for the infant's sex and gestational age compared with a reference population (2011 US birth certificate data) ${ }^{(24)}$. Cut-offs for the 10th percentiles of birth weight were determined separately for each infant sex; and within each sex, they were determined separately for each week in gestation. While birth weight curves specific to maternal race/ethnicity and parity have been used in some studies, prior studies of fish consumption and SGA have used birth weight curves specific to infant sex and gestational age only. To compare our results with prior findings, we chose to use comparable birth weight measures.

\section{Eligibility criteria}

NBDPS control participants who delivered a live-born singleton infant were eligible for inclusion in the present analyses. Mother-infant pairs were excluded if fish consumption data were missing or if the mother had type 1 or type 2 diabetes prior to pregnancy. Women with diabetes diagnosed prior to pregnancy were excluded from analyses because it is strongly associated with both preterm birth and large for gestational age ${ }^{(25,26)}$; moreover, with $<1 \%$ of births among women with type 1 diabetes ${ }^{(27)}$, there would not be an adequate number of exposed women to assess confounding. For the SGA analyses, mother-infant pairs were also excluded if the infant's birth weight or sex was not provided or if the infant's gestational age fell outside the range of gestational ages for which a 10th percentile standard was available, i.e. $<24$ or $>42$ weeks at delivery.

\section{Statistical analyses}

To facilitate comparisons with existing literature, we categorized the sixteen fish consumption categories from the FFQ as follows: less than once per month, 1-3 times per month, once per week, 2-6 times per week, and once per day or more. The following potential confounders were selected $a$ priori $^{(6,7,28,29)}$ from variables collected in the NBDPS interview and categorized as follows: maternal 
race/ethnicity (non-Hispanic white; non-Hispanic black; US-born Hispanic; foreign-born Hispanic; US-born other; foreign-born other), maternal age (16-19, 20-29, 30-39 and 40-49 years), maternal education ( $\leq 12$ years/high school or General Educational Development; $13-15$ years/ some college or associate degree; $\geq 16$ years/college degree or higher), maternal pre-pregnancy BMI $(<18.5$, $18 \cdot 5-<25 \cdot 0,25 \cdot 0-<30 \cdot 0$ and $\geq 30 \cdot 0 \mathrm{~kg} / \mathrm{m}^{2}$ ), household income (<\$US 20 000; \$US 20 000-50 000; > US \$50 000), household size (1-2 people; $3-4$ people; 5-6 people; $\geq 7$ people), smoking (no smoking in pregnancy $v$. any smoking in pregnancy), alcohol use (no alcohol use in periconceptional period $v$. alcohol use in the periconceptional period), gestational diabetes (no gestational diabetes $v$. diabetes during pregnancy), hypertension (no hypertension; hypertension with medication; hypertension without medication), parity (0, 1, 2 and $\geq 3$ prior live births), maternal height (quartiles), as well as average daily intake of energy, carbohydrates, total fat, protein, Fe and $\mathrm{Zn}$ in the year prior to pregnancy, calculated from the $\mathrm{FFQ}^{(23)}$ and divided into quartiles of intake to allow for non-linear relationships. Hispanic and other (predominantly Asian) race/ethnicity were divided into USborn and foreign-born because the rates of preterm birth and SGA differ between the groups ${ }^{(30,31)}$. Furthermore, the associations are in opposite directions, with foreignborn Asian mothers at increased risk and foreign-born Hispanic mothers at decreased risk of adverse outcomes compared with US-born mothers ${ }^{(31)}$. Non-Hispanic white and black mothers were not further divided by nativity due to the low proportion of foreign-born mothers in these groups. The distribution of potential confounders was assessed for differences across fish consumption levels by $\chi^{2}$ tests.

Crude OR (cOR) estimates for the association between each outcome and fish consumption categories were calculated by simple logistic regression. We used 95\% CI to assess whether the odds of having a preterm delivery or an SGA infant differed by level of fish intake using women who reported eating fish less than once per month or never as the reference group. We used logistic regression and the change in estimate method to identify confounders for inclusion in the adjusted model. The initial full multivariable logistic regression model contained indicator variables for four levels of fish intake and all covariates that were described above and that were associated with the outcome $(P<0.25)$ in simple logistic regression. The final model estimating adjusted OR (aOR) and $95 \%$ CI for each outcome contained the levels of fish intake and those covariates that resulted in a $10 \%$ or greater change in one of the aOR for fish consumption when they were dropped from the full model. Additional sensitivity analyses were conducted looking at the association between fish consumption and early preterm births ( $<32$ weeks and $<35$ weeks of gestation) and SGA restricted to full-term infants only.
We assessed interactions between the level of fish intake and the following covariates: maternal race/ethnicity and maternal education. Interaction terms were retained in the multivariable model if the group of interaction terms was significantly associated with the outcome (likelihood ratio test $P<0 \cdot 05$ ). We checked model fit for the final models using the Hosmer and Lemeshow goodness-of-fit test $(P<0.05$ indicating poor fit). All analyses were conducted using the statistical software package SAS version 9.4. Finally, we assessed how robust the association estimates were to unmeasured confounding by conducting a sensitivity analysis looking at the strength of association that an unmeasured confounder would need to have with both the exposure and the outcome to explain the observed association (E-value ${ }^{(32)}$. The E-value aids in assessment of causality in observational studies that may be affected by confounding by quantifying the strength of association an unmeasured confounder would need to have to explain the results: a large value implies a strongly associated unmeasured confounder would need to be present, while a small value implies a weakly associated unmeasured confounder could explain the observed association $^{(32)}$.

\section{Results}

There were 11829 control mothers included in the NBDPS with estimated due dates between 1997 and 2011, of whom 11451 (97\%) delivered singletons with a gestational age of at least 20 weeks and were eligible for inclusion in the current analyses. Mothers missing fish consumption data ( $n$ 461) or with pre-existing type 1 or type 2 diabetes ( $n$ 71) were excluded, leaving 10919 mother-infant pairs for the preterm analysis. Infants were additionally excluded from the SGA analysis if they were missing birth weight ( $n$ 145), infant sex ( $n$ 10) or had a gestational age outside the gestational age range of 24 to 42 weeks ( $n$ 48). These exclusions left 10716 motherinfant pairs for the SGA analysis. Overall, $853(7 \cdot 8 \%)$ infants were preterm and $828(7.7 \%)$ were SGA, while forty-six (0.4\%) infants were both preterm and SGA.

Women reported 3.3 servings of fish per month on average and $31.6 \%$ ( $n$ 3446) of women reported eating no fish or eating it less than once per month (Table 1). Maternal sociodemographic characteristics differed across fish consumption categories $\left(\chi^{2} P<0 \cdot 05\right.$, Table 1$)$. Women in the highest consumption group were more likely to be black, foreign-born Hispanic or foreign born-other (selfreported race as Asian, Native American or other) compared with women in the lowest consumption group, who were more likely to be white or US-born Hispanic. Women in the highest and lowest consumption groups were more likely to have a high-school education or less compared with women with moderate consumption (1-3 times per month, once per week or 2-6 times per week). Women 
Table 1 Maternal sociodemographic characteristics associated with fish consumption frequency in the National Birth Defects Prevention Study, 1997-2011

\begin{tabular}{|c|c|c|c|c|c|c|c|}
\hline & \multirow[b]{2}{*}{$n$} & \multicolumn{5}{|c|}{ Fish consumption frequency (row \%) } & \multirow[b]{2}{*}{$x^{2} P$ value } \\
\hline & & $<1 /$ month & $1-3 /$ month & 1/week & 2-6/week & $\geq 1 / d$ & \\
\hline Total & 10919 & $31 \cdot 6$ & $33 \cdot 3$ & $21 \cdot 0$ & $13 \cdot 1$ & 1.0 & \\
\hline Age at conception (years) & & & & & & & $<0.001$ \\
\hline $16-19$ & 1286 & 49.5 & $27 \cdot 6$ & 13.5 & 8.0 & 1.3 & \\
\hline $20-29$ & 5707 & $33 \cdot 5$ & 33.6 & $20 \cdot 1$ & $12 \cdot 0$ & 0.8 & \\
\hline 30-39 & 3625 & $22 \cdot 0$ & $35 \cdot 2$ & $25 \cdot 1$ & $16 \cdot 6$ & $1 \cdot 1$ & \\
\hline $40-49$ & 166 & $16 \cdot 9$ & $31 \cdot 3$ & $27 \cdot 7$ & 23.5 & 0.6 & \\
\hline Race/ethnicity & & & & & & & $<0.001$ \\
\hline White & 6373 & 34.6 & $35 \cdot 6$ & $19 \cdot 4$ & $10 \cdot 1$ & 0.3 & \\
\hline Black & 1173 & $16 \cdot 3$ & $31 \cdot 9$ & $25 \cdot 8$ & $23 \cdot 4$ & $2 \cdot 7$ & \\
\hline Hispanic, US-born & 1122 & $37 \cdot 9$ & $31 \cdot 6$ & $19 \cdot 6$ & $10 \cdot 2$ & 0.8 & \\
\hline Hispanic, foreign-born & 1502 & $26 \cdot 2$ & $30 \cdot 1$ & $25 \cdot 8$ & $16 \cdot 3$ & 1.6 & \\
\hline Other, US-born & 378 & 34.9 & 29.9 & $17 \cdot 5$ & $16 \cdot 1$ & 1.6 & \\
\hline Other, foreign-born & 323 & $25 \cdot 4$ & $21 \cdot 7$ & $21 \cdot 7$ & $26 \cdot 9$ & 4.3 & \\
\hline Education & & & & & & & $<0.001$ \\
\hline$\leq$ High school & 4409 & 38.5 & $29 \cdot 3$ & $18 \cdot 1$ & $12 \cdot 6$ & 1.5 & \\
\hline Some college & 2918 & 31.4 & $35 \cdot 2$ & $20 \cdot 3$ & $12 \cdot 4$ & 0.8 & \\
\hline College or higher & 3504 & $22 \cdot 8$ & $37 \cdot 2$ & $25 \cdot 2$ & $14 \cdot 4$ & 0.5 & \\
\hline Height $(\mathrm{cm})$ & & & & & & & 0.006 \\
\hline$\leq 158$ & 2770 & $32 \cdot 0$ & $31 \cdot 3$ & $21 \cdot 2$ & $14 \cdot 2$ & 1.4 & \\
\hline$\overline{159}-\leq 163$ & 2750 & $31 \cdot 0$ & $35 \cdot 4$ & $19 \cdot 9$ & $12 \cdot 9$ & 0.9 & \\
\hline $164-\leq 171$ & 2466 & $32 \cdot 7$ & 33.8 & $20 \cdot 7$ & $12 \cdot 0$ & 0.8 & \\
\hline$\geq 172$ & 2528 & 30.9 & $34 \cdot 3$ & $21 \cdot 2$ & $13 \cdot 1$ & 0.6 & \\
\hline Previous live births & & & & & & & $<0.001$ \\
\hline 0 & 4332 & $35 \cdot 0$ & 31.5 & $20 \cdot 0$ & $12 \cdot 6$ & 0.9 & \\
\hline 1 & 3574 & 29.6 & 35.4 & 21.3 & $12 \cdot 8$ & 1.0 & \\
\hline 2 & 1867 & $29 \cdot 8$ & 33.9 & $22 \cdot 3$ & $13 \cdot 4$ & 0.6 & \\
\hline$\geq 3$ & 1143 & $27 \cdot 7$ & $32 \cdot 9$ & $22 \cdot 0$ & $15 \cdot 8$ & 1.6 & \\
\hline Smoking during pregnancy & & & & & & & $<0.001$ \\
\hline No & 8905 & $29 \cdot 6$ & 33.7 & $22 \cdot 2$ & $13 \cdot 6$ & 1.0 & \\
\hline Yes & 1964 & $40 \cdot 6$ & $31 \cdot 8$ & $15 \cdot 6$ & $11 \cdot 0$ & 1.0 & \\
\hline Household income & & & & & & & $<0.001$ \\
\hline$<\$$ US 20000 & 3205 & $36 \cdot 2$ & 29.9 & $18 \cdot 7$ & $13 \cdot 5$ & $1 \cdot 8$ & \\
\hline \$US $20000-50000$ & 3226 & $34 \cdot 0$ & $35 \cdot 0$ & $19 \cdot 1$ & 11.4 & 0.6 & \\
\hline$>\$$ US 50000 & 3611 & $24 \cdot 1$ & $36 \cdot 1$ & $25 \cdot 3$ & $14 \cdot 2$ & 0.3 & \\
\hline $\begin{array}{l}\text { Number of people living in } \\
\text { the household }\end{array}$ & & & & & & & 0.001 \\
\hline $1-2$ & 3618 & 33.0 & $31 \cdot 6$ & $20 \cdot 8$ & 13.9 & 0.7 & \\
\hline $3-4$ & 5025 & $30 \cdot 3$ & $35 \cdot 2$ & $21 \cdot 4$ & $12 \cdot 4$ & 0.8 & \\
\hline $5-6$ & 1137 & $31 \cdot 2$ & 34.0 & $20 \cdot 3$ & $13 \cdot 3$ & $1 \cdot 1$ & \\
\hline$\geq 7$ & 251 & 31.5 & $29 \cdot 1$ & $22 \cdot 7$ & 13.9 & $2 \cdot 8$ & \\
\hline
\end{tabular}

who consumed fish 1-3 times per month, once per week or 2-6 times per week were more likely to have a college degree than women who consumed fish daily or less than once per month.

\section{Preterm birth}

The percentage of infants born preterm ranged from 6.7 to $7.6 \%$ among women eating fish once per week, 2-6 times per week or once per day or more, compared with $8.2 \%$ of infants born preterm among women reporting fish consumption less than once per month (Table 2). After assessing potential confounding (variables assessed are shown in the online supplementary material, Table S1), maternal race/ethnicity was the only variable retained in the adjusted model and no significant interactions were found $(P=0.31$ and 0.38$)$. After adjustment, we observed no association between fish consumption levels and preterm birth $(\mathrm{aOR}=0 \cdot 7-1 \cdot 0)$. Restricting to early preterm births ( $<32$ weeks and $<35$ weeks) $v$. full-term births ( $\geq 37$ weeks), results were similar in both analyses. Results for early preterm birth are presented for $<35$ weeks only due to sample size limitations (Table 2). We observed no association between fish consumption and early preterm delivery $<35$ weeks $(\mathrm{aOR}=0 \cdot 7-0 \cdot 9)$. OR were not reported for the highest consumption category because this group contained fewer than five early preterm infants.

\section{Small for gestational age}

The percentage of SGA infants among fish consumers ranged from $7 \cdot 1$ to $20.4 \%$ compared with $8.0 \%$ among women reporting fish consumption less than once per month (Table 3). After assessing potential confounding (variables assessed are shown in the online supplementary material, Table S2), the final adjusted model included 
Table 2 Crude and adjusted OR for the associations between levels of fish consumption and preterm birth $(<37$ weeks of gestational age) or early preterm birth (<35 weeks of gestational age) in the National Birth Defects Prevention Study, 1997-2011

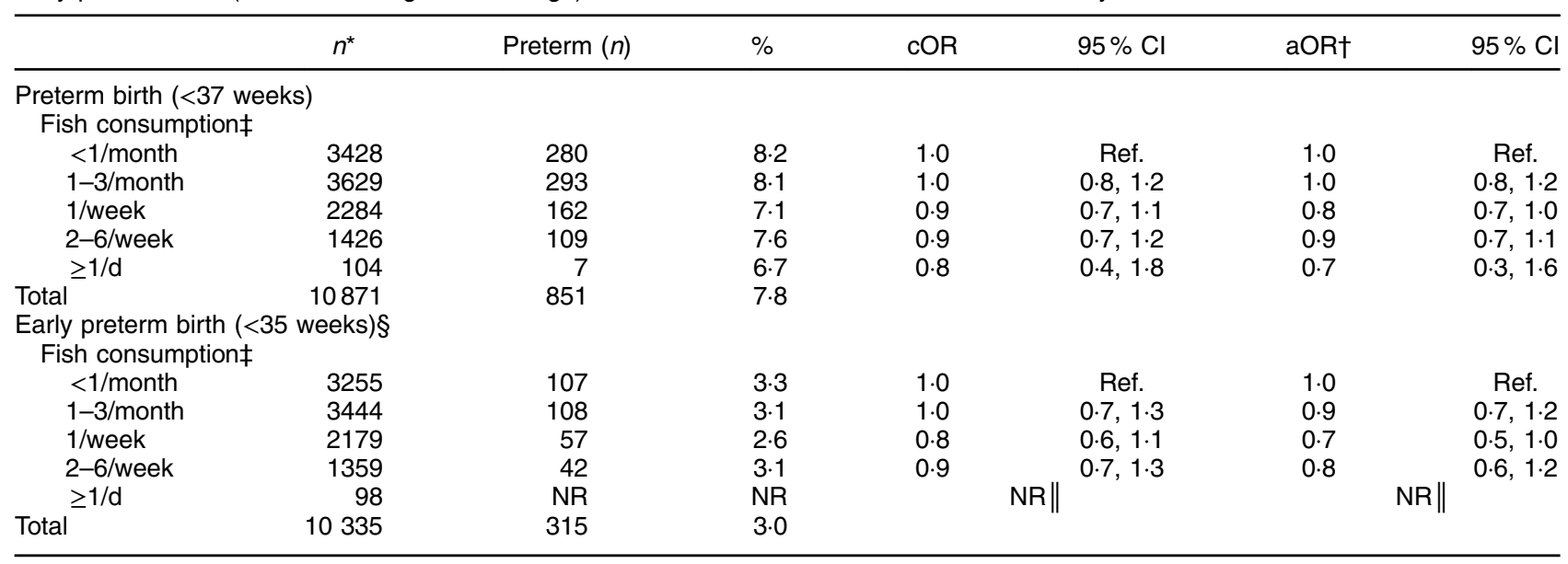

cOR, crude OR; aOR, adjusted OR; NR, not reported; Ref., reference category.

*Observations missing maternal race/ethnicity ( $n 48)$ were excluded from analysis.

†Multivariable logistic regression models adjusted for maternal race/ethnicity.

$\ddagger$ Average number of $85-140 \mathrm{~g} \mathrm{(3-5} \mathrm{oz)} \mathrm{servings} \mathrm{of} \mathrm{fish} \mathrm{eaten} \mathrm{per} \mathrm{month,} \mathrm{week} \mathrm{or} \mathrm{day} \mathrm{during} \mathrm{the} \mathrm{year} \mathrm{prior} \mathrm{to} \mathrm{pregnancy.}$

§Observations with gestational age 35-36 weeks at delivery ( $n$ 536) were excluded from analysis.

\|OR not reported due to small cell size.

Table 3 Crude and adjusted OR for the associations between levels of fish consumption and small-for-gestational-age infants (SGA; birth weight $<10$ th percentile for gestational age and infant sex) among all infants and restricted to full-term infants (37-42 weeks of gestational age) in the National Birth Defects Prevention Study, 1997-2011

\begin{tabular}{|c|c|c|c|c|c|c|c|}
\hline & $n^{\star}$ & SGA $(n)$ & $\%$ & cOR & $95 \% \mathrm{Cl}$ & $\mathrm{aOR} \dagger$ & $95 \% \mathrm{Cl}$ \\
\hline \multicolumn{8}{|c|}{ SGA, any gestational age } \\
\hline \multicolumn{8}{|c|}{ Fish consumption $\ddagger$} \\
\hline$<1 /$ month & 3338 & 267 & 8.0 & 1.0 & Ref. & 1.0 & Ref. \\
\hline $1-3 /$ month & 3562 & 254 & $7 \cdot 1$ & 0.9 & $0.7,1.1$ & 0.9 & $0.8,1 \cdot 1$ \\
\hline $1 /$ week & 2230 & 177 & 7.9 & 1.0 & $0.8,1.2$ & 1.0 & $0.8,1.2$ \\
\hline 2-6/week & 1394 & 105 & 7.5 & 0.9 & $0.7,1 \cdot 2$ & $0 \cdot 8$ & $0 \cdot 7,1 \cdot 1$ \\
\hline$\geq 1 / d$ & 103 & 21 & $20 \cdot 4$ & $2 \cdot 9$ & $1 \cdot 8,4 \cdot 8$ & $2 \cdot 1$ & $1.2,3.4$ \\
\hline Total & 10627 & 824 & $7 \cdot 8$ & & & & \\
\hline \multirow{2}{*}{\multicolumn{8}{|c|}{ SGA, full-term infants (37-42 weeks)§ }} \\
\hline & & & & & & & Fish consumption $\ddagger$ \\
\hline$<1 /$ month & 3069 & 249 & $8 \cdot 1$ & 1.0 & Ref. & 1.0 & Ref. \\
\hline $1-3 /$ month & 3274 & 240 & 7.3 & 0.9 & $0.7,1.1$ & 0.9 & $0.8,1 \cdot 1$ \\
\hline $1 /$ week & 2071 & 169 & 8.2 & 1.0 & $0.8,1.2$ & 1.0 & $0.8,1 \cdot 2$ \\
\hline 2-6/week & 1291 & 99 & $7 \cdot 7$ & 0.9 & $0.7,1.2$ & 0.8 & $0.7,1.1$ \\
\hline$\geq 1 / d$ & 96 & 21 & 21.9 & 3.2 & $1 \cdot 9,5 \cdot 2$ & $2 \cdot 2$ & $1 \cdot 3,3 \cdot 6$ \\
\hline Total & 9801 & 778 & 7.9 & & & & \\
\hline
\end{tabular}

cOR, crude OR; aOR, adjusted OR; Ref., reference category.

*Observations missing birth weight $(n 145)$, infant sex $(n 10)$, with gestational age outside the range $(<24$ or $>42$ weeks) with reference values $(n 48)$ or missing maternal race/ethnicity or maternal education $(n 89)$ were excluded from analysis.

†Multivariable logistic regression model adjusted for maternal race/ethnicity and maternal education.

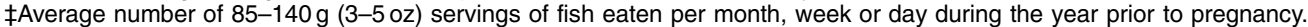

$\S$ Observations with gestational age $<37$ weeks at delivery ( $n$ 826) were excluded from analysis.

maternal race/ethnicity and maternal education and no significant interactions were found between fish consumption and either covariate $(P=0.69$ and 0.99$)$. Adjusted odds of having an SGA infant for women who ate fish 1-3 times per month $(\mathrm{aOR}=0 \cdot 9 ; 95 \%$ CI $0 \cdot 8,1 \cdot 1)$, once per week ( $\mathrm{aOR}=1 \cdot 0 ; 95 \% \mathrm{CI} 0 \cdot 8,1 \cdot 2)$ or $2-6$ times per week $(\mathrm{aOR}=0.8 ; 95 \% \mathrm{CI} 0.7,1.1)$ were not significantly different compared with women who ate fish less than once per month (Table 3). Consuming fish once per day or more was associated with increased odds of having an SGA infant $(\mathrm{aOR}=2 \cdot 1 ; 95 \% \mathrm{CI} 1 \cdot 2,3 \cdot 4) \mathrm{com}-$ pared with fish consumption less than once per month. Adjustment for confounders had the greatest impact in the highest consumption category, lowering the OR from $2 \cdot 9$ to $2 \cdot 1$ (Table 3 ).

Restricting the SGA analysis to term deliveries ( $\geq 37$ weeks) resulted in nearly identical estimates as the analysis of all SGA births. The highest fish consumption 
level was associated with elevated odds of having an SGA infant (aOR $=2 \cdot 2 ; 95 \%$ CI 1.3, 3.6; Table 3). aOR for the other consumption categories were nearly identical to estimates for the full sample and ranged from 0.8 to $1 \cdot 0$.

Based on the sensitivity analysis calculating the E-value of the robustness to unmeasured confounding, an unmeasured confounder would have to be associated with both the outcome and the exposure by a ratio of 3.6 above and beyond adjustment for the measured confounders to fully explain the observed $2 \cdot 1$-fold higher odds of SGA among daily fish consumers. An unmeasured confounder associated with both the exposure and outcome by a ratio of 1.7 or higher above and beyond the measured confounders could move the confidence interval to include the null.

\section{Discussion}

Our finding of an average of 3.3 servings (85-140g (3-5 oz)) of fish per month is similar to other studies of US women that reported fish consumption of $85-100 \mathrm{~g}$ $(3 \cdot 0-3 \cdot 5 \mathrm{oz})$ per week ${ }^{(33,34)}$. A higher proportion of women in the present study reported consuming fish less than once per month (31.6\%) than in European studies, where 8-18\% of women reported no fish consumption ${ }^{(8,9,16)}$.

A recent meta-analysis that pooled data from nineteen European birth cohorts found an $11-13 \%$ reduction in preterm birth among women eating fish twice per week or more compared with women eating fish once per week or less ${ }^{(12)}$. Studies that have categorized fish consumption in a similar manner to our study reported OR for preterm birth of 0.84 for twice per week $v$. once per week or less ${ }^{(13)}$ and 0.65 for twice per week or more $v$. less than once per month ${ }^{(9)}$. While our association estimates of a 10-30\% decrease in the odds of preterm birth among women eating fish twice per week or more were similar in magnitude to these European studies, our study lacked the precision needed to find an association of this magnitude. In contrast to most of the previous studies, one recent US study reported an increased risk of preterm birth among women consuming lean fish more than once per week ${ }^{(18)}$. We did not observe an increased risk of preterm birth among high consumers in our study; however, we were unable to evaluate lean fish consumption specifically.

Women reporting daily fish consumption had twice the odds of having an SGA infant compared with women who reported eating fish less than once per month; however, this estimate was based on twenty-one SGA infants and only a small proportion of women reported consumption levels this high. These findings should be replicated in future studies by including a high consumption category. Several prior studies have found an elevated risk of SGA among women consuming high levels of fatty fish ${ }^{(16)}$, shellfish (oysters, mussels, shrimp, prawns, lobster and (crab) ${ }^{(9)}$, crustaceans (a component of shellfish: shrimp, prawns, lobster and crab) ${ }^{(17)}$ and canned tuna ${ }^{(17)}$. The aOR estimates for SGA among women consuming shellfish, crustaceans and canned tuna twice per week or more were of similar magnitude to our findings ( $\mathrm{aOR}=2 \cdot 14$, $2 \cdot 45$ and 2.49 , respectively) ${ }^{(9,17)}$. As shellfish and canned tuna are among the most commonly eaten fish types in the $\mathrm{USA}^{(3)}$, they may also have been the most commonly eaten types of fish among the women in our study who reported daily consumption of fish.

Evidence of a relationship between high maternal fish consumption and decreased birth weight has also been previously reported in the USA. Mohanty et al. used a different outcome measure, low birth weight $(<2500 \mathrm{~g})$, and reported a $2 \cdot 2$-fold higher risk among women consuming lean fish more than once per week compared with non-consumers ${ }^{(21)}$. While we were unable to evaluate lean fish consumption specifically and replicate this finding in our study, lean fish, including canned light tuna, breaded fish products and catfish, are also commonly eaten species in the $\mathrm{USA}^{(3)}$.

Our study was limited by collecting fish consumption using a single question in the FFQ. A previous study demonstrated that a one-item FFQ about fish consumption correlated more closely with plasma DHA concentrations and comparably correlated with methylmercury intake compared with a four- or thirty-six-item $\mathrm{FFQ}^{(35)}$. Longer FFQ were found to provide no advantage over one-item FFQ in ranking intakes of fish, DHA and methylmercury $^{(35)}$. As in many previous studies, we were unable to assess which types of fish were consumed by women in the highest consumption group and how preparation methods may have impacted nutrient and contaminant contents. Since both fish and shellfish are sources of nutrients and possibly contaminants, more detailed consumption and preparation data should be collected in future studies. Additionally, since we performed a secondary analysis of data from a study of birth defects in which the critical window for development of the outcome occurs early in pregnancy, the present study queried average fish consumption during the year prior to conception. Prior studies of fish consumption and pregnancy outcomes vary widely in the timing of collection of fish consumption data, with studies collecting FFQ data in the first trimester ${ }^{(9,18,21)}$, second trimester ${ }^{(11,13,14,16)}$, third trimester $^{(8)}$, or at multiple points in pregnancy ${ }^{(10,15,17)}$. It is possible that some women in the present study may have changed their consumption habits during pregnancy. Recently Razzaghi and Tinker reported no differences in seafood consumption between pregnant and nonpregnant US women using both detailed $30 \mathrm{~d}$ fish intake data and $24 \mathrm{~h}$ dietary recalls collected in the National Health and Nutrition Examination Survey from 1999 to $2006^{(36)}$. Additionally, women were asked to recall their average consumption during the year prior to pregnancy, which may have introduced recall bias. Finally, there may be residual confounding or bias affecting our observed 
associations. Our calculated E-value of 3.6 indicates that only a strong unmeasured confounder could fully explain the association observed between daily fish consumption and SGA. None of the potential confounders we did evaluate were associated this strongly with the outcome. While our results are fairly robust to unmeasured confounding and we assessed a number of potential confounders in our analyses, we cannot rule out the possibility that women who reported daily fish consumption may have had other co-occurring health behaviours or dietary patterns that were not assessed and may be driving the results.

The strengths of our study include the large sample size and the diverse study population from multiple regions across the USA. We assessed the association between high fish consumption and SGA infants. Previous studies that have grouped fish consumption by quartiles may have masked elevated risk in high consumers by grouping them with moderate consumers. We would not have observed an association between high fish consumption and SGA if we had grouped women consuming fish 2-6 times per week with the highest consumers (once per day or more). Additionally, as our sample was based on NBDPS controls, none of the infants in our analyses had chromosomal abnormalities or other major structural birth defects, which can impact gestational age at birth and birth weight, and we were able to assess potential confounding by other dietary components calculated from the FFQ.

To the best of our knowledge, the present study is the first US one to observe an elevated risk of SGA among women reporting daily fish consumption. The elevated risk of SGA we observed among high fish consumers should be confirmed and future studies should collect more detailed data on fish consumption to investigate whether a specific component or contaminant is associated with SGA. Currently the US Food and Drug Administration recommends that when eating fish from commercial sources, women of childbearing age and pregnant women should eat 2-3 servings of fish lower in methylmercury ('best choices') or 1 serving of fish with moderate methylmercury content ('good choices'), while avoiding fish with high mercury content ${ }^{(37)}$. Our results are consistent with the Food and Drug Administration's recommendation in suggesting that, with respect to the risk of preterm birth and SGA, moderate intake of fish may be beneficial and high intake may be harmful. These results add to the body of evidence that women of childbearing age should be counselled on appropriate fish consumption for a healthy pregnancy.

\section{Acknowledgements}

Financial support: This publication was supported in part through a cooperative agreement (U01DD000494) between the Centers for Disease Control and Prevention (CDC) and the Texas Department of State Health Services (DSHS). Its contents are solely the responsibility of the authors and do not necessarily represent the official views of the CDC or the Texas DSHS. Conflict of interest: None. Authorship: R.H.B. was involved in study design, literature review, data analysis and manuscript writing. L.E.M., M.A.C., T.A.R., S.A.C. and A.P.C. were involved in study design and manuscript revisions. A.T.H. replicated analyses and was involved in manuscript revisions. D.L. supervised statistical methods and reviewed the manuscript. D.K.W. was the senior investigator involved in study design, analysis and manuscript revisions. Ethics of buman subject participation: This study was conducted according to the guidelines laid down in the Declaration of Helsinki and all procedures involving human subjects were approved by the Institutional Review Board for each centre. Approval for this secondary analysis was granted by The University of Texas Health Science Center at Houston Committee for the Protection of Human Subjects.

\section{Supplementary material}

To view supplementary material for this article, please visit https://doi.org/10.1017/S1368980018002641

\section{References}

1. Coletta JM, Bell SJ \& Roman AS (2010) Omega-3 fatty acids and pregnancy. Rev Obstet Gynecol 3, 163-171.

2. Jensen CL (2006) Effects of $n-3$ fatty acids during pregnancy and lactation. Am J Clin Nutr 83, 6 Suppl., 1452S-1457S.

3. Mahaffey KR, Clickner RP \& Jeffries RA (2008) Methylmercury and omega- 3 fatty acids: co-occurrence of dietary sources with emphasis on fish and shellfish. Environ Res 107, 20-29.

4. Kris-Etherton PM, Harris WS \& Appel LJ (2002) Fish consumption, fish oil, omega-3 fatty acids, and cardiovascular disease. Circulation 106, 2747-2757.

5. Oken E \& Bellinger DC (2008) Fish consumption, methylmercury and child neurodevelopment. Curr Opin Pediatr 20, 178-183.

6. Institute of Medicine (2007) Preterm Birth: Causes, Consequences, and Prevention. Washington DC: The National Academies Press; available at http://www.nap.edu/catalog/ 11622.html.

7. Centers for Disease Control and Prevention (2006) QuickStats: Percentage of small-for-gestational-age births, by race and Hispanic ethnicity - United States, 2005. https://www.cdc. gov/mmwr/preview/mmwrhtml/mm5750a5.htm (accessed October 2017).

8. Rogers I, Emmett P, Ness A et al. (2004) Maternal fish intake in late pregnancy and the frequency of low birth weight and intrauterine growth retardation in a cohort of British infants. J Epidemiol Community Health 58, 486-492.

9. Guldner L, Monfort C, Rouget F et al. (2007) Maternal fish and shellfish intake and pregnancy outcomes: a prospective cohort study in Brittany, France. Environ Health 6, 33.

10. Olsen SF, Osterdal ML, Salvig JD et al. (2006) Duration of pregnancy in relation to seafood intake during early and 
mid pregnancy: prospective cohort. Eur J Epidemiol 21, 749-758.

11. Klebanoff MA, Harper M, Lai Y et al. (2011) Fish consumption, erythrocyte fatty acids, and preterm birth. Obstet Gynecol 117, 1071-1077.

12. Leventakou V, Roumeliotaki T, Martinez D et al. (2014) Fish intake during pregnancy, fetal growth, and gestational length in 19 European birth cohort studies. Am J Clin Nutr 99, 506-516.

13. Haugen M, Meltzer HM, Brantsaeter AL et al. (2008) Mediterranean-type diet and risk of preterm birth among women in the Norwegian Mother and Child Cohort Study (MoBa): a prospective cohort study. Acta Obstet Gynecol Scand 87, 319-324.

14. Brantsaeter AL, Englund-Ogge L, Haugen M et al. (2017) Maternal intake of seafood and supplementary long chain $n-3$ poly-unsaturated fatty acids and preterm delivery. $B M C$ Pregnancy Childbirth 17, 41.

15. Olsen SF \& Secher NJ (2002) Low consumption of seafood in early pregnancy as a risk factor for preterm delivery: prospective cohort study. BMJ 324, 1-5.

16. Halldorsson TI, Meltzer HM, Thorsdottir I et al. (2007) Is high consumption of fatty fish during pregnancy a risk factor for fetal growth retardation? A study of 44,824 Danish pregnant women. Am J Epidemiol 166, 687-696.

17. Mendez MA, Plana E, Guxens M et al. (2010) Seafood consumption in pregnancy and infant size at birth: results from a prospective Spanish cohort. J Epidemiol Community Health 64, 216-222.

18. Mohanty AF, Siscovick DS, Williams MA et al. (2016) Periconceptional seafood intake and pregnancy complications. Public Health Nutr 19, 1795-1803.

19. Food and Agriculture Organization of the United Nations (2016) The State of World Fisheries and Aquaculture, 2016. http://www.fao.org/3/a-i5555e.pdf (accessed July 2017).

20. Oken E, Kleinman KP, Olsen SF et al. (2004) Associations of seafood and elongated $n-3$ fatty acid intake with fetal growth and length of gestation: results from a US pregnancy cohort. Am J Epidemiol 160, 774-783.

21. Mohanty AF, Thompson ML, Burbacher TM et al. (2015) Periconceptional seafood intake and fetal growth. Paediatr Perinat Epidemiol 29, 376-387.

22. Yoon PW, Rasmussen SA, Lynberg MC et al. (2001) The National Birth Defects Prevention Study. Public Health Rep 116, 32-40.

23. Willett WC, Sampson L, Stampfer MJ et al. (1985) Reproducibility and validity of a semiquantitative food frequency questionnaire. Am J Epidemiol 122, 51-65.

24. Duryea EL, Hawkins JS, McIntire DD et al. (2014) A revised birth weight reference for the United States. Obstet Gynecol 124, 16-22.
25. Colstrup M, Mathiesen ER, Damm P et al. (2013) Pregnancy in women with type 1 diabetes: have the goals of St. Vincent declaration been met concerning foetal and neonatal complications? J Matern Fetal Neonatal Med 26, $1682-1686$.

26. McCance DR (2015) Diabetes in pregnancy. Best Pract Res Clin Obstet Gynaecol 29, 685-699.

27. Peng TY, Ehrlich SF, Crites Y et al. (2017) Trends and racial and ethnic disparities in the prevalence of pregestational type 1 and type 2 diabetes in Northern California: 1996-2014. Am J Obstet Gynecol 216, 177.e1-e8.

28. Goldenberg RL, Culhane JF, Iams JD et al. (2008) Epidemiology and causes of preterm birth. Lancet $\mathbf{3 7 1}$, 75-84.

29. Carmichael SL, Yang W, Shaw GM et al. (2013) Maternal dietary nutrient intake and risk of preterm delivery. $A m \mathrm{~J}$ Perinatol 30, 579-588.

30. Gould JB, Madan A, Qin C et al. (2003) Perinatal outcomes in two dissimilar immigrant populations in the United States: a dual epidemiologic paradox. Pediatrics 111, e676-e682.

31. Gagnon AJ, Zimbeck M, Zeitlin J et al. (2009) Migration to western industrialised countries and perinatal health: a systematic review. Soc Sci Med 69, 934-946.

32. VanderWeele TJ \& Ding P (2017) Sensitivity analysis in observational research: introducing the E-value. Ann Intern Med 167, 268-274.

33. US Department of Agriculture \& US Department of Health and Human Services (2010) Dietary Guidelines for Americans, 2010, 7th ed, Washington, DC: US Government Printing Office; available at https://health.gov/dietary guidelines/dga2010/dietaryguidelines2010.pdf

34. Papanikolaou Y, Brooks J, Reider C et al. (2014) US adults are not meeting recommended levels for fish and omega-3 fatty acid intake: results of an analysis using observational data from NHANES 2003-2008. Nutr J 13, 31.

35. Oken E, Guthrie LB, Bloomingdale A et al. (2014) Assessment of dietary fish consumption in pregnancy: comparing one-, four-, and thirty-six-item questionnaires. Public Health Nutr 17, 1949-1959.

36. Razzaghi H \& Tinker SC (2014) Seafood consumption among pregnant and non-pregnant women of childbearing age in the United States, NHANES 1999-2006. Food Nutr Res 11, 58 .

37. US Food and Drug Administration \& US Environmental Protection Agency (2017) Eating Fish: What Pregnant Women and Parents Should Know. https://www.fda.gov/downloads/ Food/FoodborneIllnessContaminants/Metals/UCM536321.pdf (accessed July 2017). 